Research Article

\title{
Translation and Validation of "Brief Bedside Dysphagia Screening Test" in a Greek Sample of Neurological Patients
}

Sophia Demeneopoulou 1, Marianna Mantzorou 2, Petra Mandysova ${ }^{3}$, Nikoletta Margari ${ }^{2}$, Eleni Dokoutsidou ${ }^{2}$, Aggeliki Tsiligianni ${ }^{4}$, Chrysoula Tsiou ${ }^{2}$, Theodoula Adamakidou ${ }^{2, *}$

1. "Help at Home" Service, Municipality of Saronikos, Sakka Keratea, PO box 50086, PC 19001, Athens, Greece; E-Mail: ertileraris@yahoo.gr

2. Department of Nursing, University of West Attica, Agiou Spiridonos, 12243 Egaleo, Athens, Greece; E-Mails: mantzorou@uniwa.gr; nmargari@uniwa.gr; edokout@uniwa.gr; ctsiou@uniwa.gr; thadam@uniwa.gr; adamakidou@yahoo.gr

3. Department of Nursing, Faculty of Health Studies, University Pardubice, Studentská 95, 53210 Pardubice, Czech Republic; E-Mail: petra.mandysova@upce.cz

4. General University Hospital of Larisa, 3 Adrianou, 41221 Larisa, Greece; E-Mail: atsiligianni81@hotmail.com

* Correspondence: Theodoula Adamakidou; E-Mails: thadam@uniwa.gr; adamakidou@yahoo.gr

Academic Editor: David G Smithard

Collection: Dysphagia in the Elderly

OBM Geriatrics

2021, volume 5 , issue 2

doi:10.21926/obm.geriatr.2102165
Received: October 09, 2020

Accepted: March 23, 2021

Published: April 02, 2021

\begin{abstract}
Dysphagia may become a threatening condition if not diagnosed and treated at an early stage. Healthcare professionals require simple, rapid, and reliable tools for the immediate evaluation of dysphagia in their daily clinical practice. The aim of the present study was the translation and validation of the "Brief Bedside Dysphagia Screening Test-Revised (BBDST-R)" tool in the Greek language in a sample of patients with neurological disorders. The present study was designed as a cross-sectional study conducted with a convenience sample of 80 patients. The BBDST-R tool contains eight items, and a positive response to an item implies that the patient
\end{abstract}

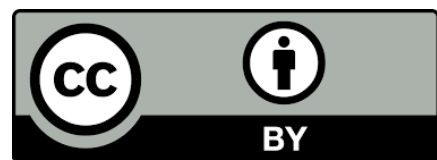

(C) 2021 by the author. This is an open access article distributed under the conditions of the Creative Commons by Attribution License, which permits unrestricted use, distribution, and reproduction in any medium or format, provided the original work is correctly cited. 
might have dysphagia. The bedside assessment using the BBDST-R tool was conducted by a researcher previously trained for the tool usage. The translation and validation were conducted by following the relevant international standards. The researcher's final assessment concerning the presence or absence of dysphagia was compared to the medical diagnosis as determined by a physician, which was set as a benchmark. The evaluation of BBDST-R was conducted by researching its diagnostic accuracy according to the guidelines for measures of diagnostic accuracy. Internal consistency was determined through Cronbach's Alpha. The English version of the BBDST-R tool has been reliably translated into the Greek language. The prevalence of dysphagia in our sample was $62.5 \%$ (95\% Cl: $50 \%-72 \%$ ). The observed high sensitivity [Sen $=98 \% / 87 \%-99 \%(95 \% \mathrm{CI})$ ] and high negative predictive value [NPV $=89 \% / 77 \%-95 \%(95 \% \mathrm{Cl})]$ indicated high validity of the screening tool the acceptable levels of $>75 \%$ even for $95 \% \mathrm{Cl}$. Cronbach's Alpha internal consistency index for the 8 items of this tool presented an almost excellent value of 0.859 . The results of the present study indicated that BBDST- $R$ is a reliable and valid tool for application in the assessment of dysphagia in patients admitted in Greek hospitals and community-based individuals.

\section{Keywords}

Dysphagia; dysphagia screening; brief bedside dysphagia screening test-revised; validation; translation; neurological disorders

\section{Introduction}

Nurses have to frequently treat and care for patients in the community or institutional settings who might develop or already have life-threatening conditions such as dysphagia. Simple, although reliable tools are required to conduct the first assessment and subsequent further investigation of the situation.

The term "dysphagia ", which is constituted of the prefix "dys" and the root "fag" having origins in the ancient Greek language, refers to a disorder involving difficulty in swallowing.

Dysphagia is quite common, with several studies reporting a prevalence of dysphagia in nursing homes to be in the range of $12.8 \%-52.7 \%$ [1-3]. In the community, the prevalence of swallowing difficulties is $20.1 \%$ among healthy elderly [4] and $5 \%-72 \%$ among the elderly [5]. A systematic review by Takizawa et al. (2016) reported that dysphagia was diagnosed in $8.1 \%-80 \%$ of stroke patients, $27 \%-30 \%$ of brain injury patients, $11 \%-81 \%$ of Parkinson's disease patients, and $91.7 \%$ of older persons with community-acquired pneumonia due to impaired breathing mechanisms and swallowing [6]. Dysphagia is usually attributed to advanced age, clinical diseases, and frailty that leads to self-care deficits [5].

Dysphagia is attributed to various causes, such as mechanical, neurological, and psychogenic conditions [7]. Moreover, dysphagia is often under-diagnosed and under-treated [7] and is, therefore, associated with an increased risk of malnutrition, dehydration, pneumonia [8], and mortality [2]. Interdisciplinary collaboration and appropriate tools are required for the diagnosis, management, and prevention of dysphagia-associated complications. Nurses play an essential role 
as the member of the interdisciplinary team and, therefore, should have access to all the necessary tools $[9,10]$.

Dysphagia is commonly assessed using imaging methods, particularly in acute care settings. A variety of non-imaging methods [11] based on questionnaires and swallowing assessment using semi-solid or liquid food are also available for clinical bedside assessment of dysphagia by health care professionals [12]. The non-imaging methods are convenient for use in acute, community, or nursing home settings where routine reassessment is vital for maintaining the health and safety of the patient.

Unlike the situation in other countries, it is not common for the nurses in Greece to be involved in the assessment of dysphagia, which is usually conducted by a physician or a speech therapist using clinical bedside assessment tools and imaging methods. However, the involvement of nurses in this evaluation is important owing to their frequent contact with the patient, the role and responsibility they have in patient feeding, the holistic evaluation they perform, and the care they deliver to the patients. The fact that there is a nursing diagnosis of "impaired swallowing" reported by NANDA International $[13,14]$ further supports the requirement of nurses' involvement in dysphagia assessment. Ideally, dysphagia assessment requires a multidisciplinary approach, and since nurses are the first line of members in the health care team to interact with the patients, they could perform the basic dysphagia screening for the patients, based on the results of which, a further detailed clinical assessment by the physician and/or speech therapist could be completed.

The validity and reliability of the swallowing screening tools for dysphagia used by nurses is a research topic that has gained great attention in healthcare systems [11].

The Brief Bedside Dysphagia Screening Test-Revised (BBDST-R) is a convenient-to-use tool for healthcare professionals to perform the first assessment of dysphagia. BBDST-R is a previously validated screening tool [15], with high sensitivity [Sen $=95.5 \% / 84.9 \%-98.7 \%(95 \% \mathrm{CI})$ ] and high negative predictive value [NPV $=88.9 \% / 67.2 \%-99.9 \%(95 \% \mathrm{CI})$ ]. However, the results obtained using the tool vary among different nurses who perform the tests [16]. The researchers have concluded that such differences are expected, and with proper training in the use of the tool, these differences would not exert a negative impact on the reliability of the tool.

The aim of the present study was the translation and validation of the Brief Bedside Dysphagia Screening Test-Revised (BBDST-R) in the Greek language in a sample of patients with neurological disorders.

\section{Materials and Methods}

\subsection{Design}

The present study was designed as a cross-sectional study conducted with a convenience sample of 80 patients having neurological health conditions who were treated in the Neurological Clinic of a large hospital in Athens and those who were treated in a private rehabilitation center. The study duration was from March 2016 to August 2016.

\subsection{Research Tools}

The research tools employed in the present study were: a) a questionnaire containing items regarding the patients' demographic and clinical characteristics, such as gender, age, marital status, 
educational level, occupation, place of residence, neurological diseases, and comorbidities, and b) the BBDST-R which was originally developed and validated for the Czech population. -The tool was used in a patient sample-with various neurological conditions, with the stroke patients constituting the largest subgroup $[15,17]$.

The BBDST-R comprises the following eight items: BBDST-R_1 presence of voluntary cough, BBDST-R_2 ability to clench the teeth, BBDST-R_3 symmetry and strength of the tongue, BBDST-R_4 symmetry and strength of the facial muscles, BBDST-R_5 symmetry and strength of shoulder shrug, BBDST-R_6 dysarthria, BBDST-R_7 aphasia, and BBDST-R_8 cough after consumption of a thick liquid.

The screening test was simple and could be performed by a healthcare professional. The response to the evaluation in each item of the questionnaire could be either YES or NO, i.e., the patient may or may not be displaying what each parameter indicated. The items that were normal received a score of 0 , while the items that were abnormal received a score of 1 . If the total score was 0 , the test was negative (i.e., the patient was un likely to have dysphagia), while in the case of the final score being $\geq 1$, the test was considered positive (i.e., the patient might be having dysphagia).

The researchers [15] indicated that it is a short and reliable tool that could be used by all health professionals who are properly trained for its use.

Mrs. Petra Mandysova, who developed the BBDST-R tool originally, was contacted, and relevant permission for using the tool in the present study was obtained $[15,16]$. The translation of the English version of BBDST-R was conducted in accordance with the international standards [18], such that the accuracy of the parameters concerned with the diagnostic purpose was not altered.

The basic principles of BBDST-R were presented by its developer in the Greek Postgraduate Program "Neurological Disorders - Evidence- Based Practice" of the Technological Educational Institute (TEI) of Athens, and the evaluation of the tool and the role of nurses in the diagnosis of dysphagia were, meanwhile, performed by Greek postgraduate nursing students [14].

\subsection{Data Collection}

The principal researcher performed the bedside assessment using the BBDST-R tool on the 80 patients with neurological disorders included in the present study.

The inclusion criteria used were: a) diagnosis of a neurological disorder [stroke, dementia, multiple sclerosis (MS), Parkinson's, myasthenia, myopathy, Guillain Barre], b) adequate level of consciousness and the ability to respond to simple commands, c) over two days of hospital stay, and d) informed consent provided for participation in the present study (by patients or their caregivers).

The exclusion criteria were: a) patients with severe mental disability, b) patients with changes in the anatomy of the mouth, pharynx, and larynx, resulting from an accident or surgery, c) patients who did not speak the Greek language, and d) patients with difficulty in eating due to anorexia or any other psychological problem.

Particular emphasis was placed on preparing the patient for the BBDST-R evaluation. All patients were subject to the participation criteria for the present study, and if the patient fulfilled the criteria, he/she was informed regarding the research and asked to provide consent for participation. Subsequently, all the necessary materials were placed on the bedside table of the patient. The patient and/or his/her caregiver were then informed regarding the procedure to be followed. Oral 
hygiene was performed, and the patient was asked to be in a sitting position (90 degrees) for the evaluation.

A crucial parameter was the preparation of the viscous liquid, for which the thick and convenient instant coagulation factors were considered. The consistency of the prepared liquid was puddinglike, which would fall- off the spoon in chunks. The liquid preparation was followed by the evaluation of the patient for each parameter. The researcher screened each patient and prepared the viscous fluid according to the instructions provided in a previously-conducted training program led by the original developer of the tool [14].

The evaluation process included the patient's assessment for each of the eight items in the tool. If, at any stage of the screening, an abnormal finding was detected, then the test result was considered to be positive, implying that the patient, according to the test, was likely to be having dysphagia, and the evaluation process was considered to be complete by itself.

The test result was then compared to the physician's diagnosis regarding the presence or absence of dysphagia in each patient (obtained from the medical records of the patients). The physician's diagnosis was based on endoscopic examination, clinical examination, and/or an appropriate diagnostic tool, and the assessment was regarded as the benchmark against which the principal researcher's assessment was compared.

\subsection{Translation}

The translation of BBDST-R was performed according to "The minimal Translation criteria" [18]. Two independent native English speakers translated the original English tool into the Greek language, which was followed by the comparison of these two translations by a third individual who arrived at the final consensus. Consequently, this version of the tool was reverse- translated into the English language. Next, the original and the reverse-translated versions of the tool were compared by the research team, and the final translation was sent to the original developer of the tool for approval.

\subsection{Ethics Statement}

The study was conducted in accordance with the Declaration of Helsinki and was approved by the ethics committee of two institutions. In addition, permission for using the tool in the present study was obtained from the original developer of the tool. All participants were informed regarding the purpose of the study, and written consent for participation was obtained from all of them.

\subsection{Statistical Analysis}

The value of any diagnostic test is quantifiable through the evaluation of the diagnostic accuracy of that test, i.e., the ability of the test to distinguish between the disease and non-disease (healthy patient) conditions.

The basic measures for diagnostic tests, such as sensitivity (Sen) and specificity (Spe), were utilized as described by Altman \& Bland [19], Glas et al. [20], and Šimundić [21]. These measures are derived from the four possible outcomes of any diagnostic test, which are then formulated in a $2 \times$ 2 contingency table. The following outcomes are possible: FN-false negative; FP-false positive; TN- 
true negative; and TP-true positive. With these outcomes, multiple parameters could be formulated, as presented in Table 1, along with the ROC curve, Diagnostic odds ratio (DOR), and others [20, 21].

Table 1 Basic measures of diagnostic accuracy (adapted from Molina [22]).

\begin{tabular}{|c|c|c|}
\hline & Healthy & Sick \\
\hline Positive & TP & FP \\
\hline Negative & FN & TN \\
\hline \multicolumn{3}{|c|}{$\begin{array}{l}\text { TP =True Positive, FP =False Positive, FN =False Negative, } T N=\text { True Negative, Sen =Sensitivity } \\
=T P /(T P+F N) \text {, Spe }=\text { Specificity }=T N /(T N+F P), P P V=\text { Positive Predictive Value }=T P /(T P+F P), N P V= \\
\text { Negative Predictive Value =TN } /(T N+F N), P L R=\text { Positive Likelihood Ratio =Se/(1-Sp), NLR } \\
=\text { Negative Likelihood Ratio =(1-Se)/Sp, Al =Accuracy Index =(TP+TN)/(TP+TN+FP+NF), Yi } \\
=\text { Youden's Index =Se+Sp-1 }\end{array}$} \\
\hline
\end{tabular}

A simple dysphagia test, such as that conducted using BBDST-R, should have high Sen and a high negative predictive value (NPV) to be considered highly valid [19].

The expected Sen value was set at $95 \%$, with the lower confidence limit for $\mathrm{Cl}=95 \%$ at 0.75 . The minimum acceptable sample size, as reported by Flahault et al. [23] and corrected by Chu and Cole [24], was $N=70-80$ patients, for a disease prevalence of $<50 \%$.

Finally, each item was tested against the patient's medical diagnosis using the association coefficient $\phi$, while $x^{2}$ was used for testing the final BBDST-R result against the diagnosis, and the test's internal consistency was measured in terms of Cronbach's Alpha.

IBM SPSS Ver 19.0, Microsoft Excel, and Clinical Calculator 1 (available at vassarstats.net) were employed for conducting the statistical analysis.

\section{Results}

A total of 103 patients were invited to participate in the present study, among which 14 patients refused to participate, nine patients did not complete the procedure for various reasons, such as withdrawal from the study, discharge, transfer to another clinic, or death, and the remaining 80 patients completed the test.

The majority of the patients (53.8\%) belonged to the age range of $81-90$ years, and $53.8 \%$ of all the participants were women. The main neurological disease (65\% of patients) was stroke, while the most common (23.8\%) coexisting condition was cervical spine disorder. Among all participants, 41.3\% were widowed, $58.8 \%$ were retired, and $31.3 \%$ had a non-paid individual as their main caregiver. The demographic and clinical data of the sample are presented in Table 2.

Table 2 Demographic and clinical characteristics of the sample.

\begin{tabular}{llll}
\hline & & N & $\%$ \\
\hline Gender & Male & 37 & 46.3 \\
& Female & 43 & 53.8 \\
Age & $40-50$ & 2 & 2.5 \\
& $51-60$ & 5 & 6.3
\end{tabular}




\begin{tabular}{llll} 
& $61-70$ & 13 & 16.3 \\
Neurological & $71-80$ & 17 & 21.3 \\
disease & $81-90$ & 43 & 53.8 \\
& Stroke & 52 & 65.0 \\
& Parkinson disease & 5 & 6.3 \\
& Multiple sclerosis & 1.3 \\
Education & Myopathy & 1 & 1.3 \\
& Dementia & 1 & 18.8 \\
& Other & 15 & 7.5 \\
Primary school & High school & 6 & 30.0 \\
& Higher education & 24 & 41.3 \\
& Msc/Phd & 33 & 21.3 \\
& No & 17 & 7.5 \\
& Cervical spine disorders & 6.5 \\
& Brain injury & 6 & 23.8 \\
& Chronic dysphagia due to aging & 2 & 2.5 \\
& Depression & 7 & 8.8 \\
& Anorexia nervosa & 1 & 1.3 \\
& Gastroesophageal reflux & 5 & 6.3 \\
& Cancer & 7 & 8.8 \\
& Respiratory diseases & 8 & 10.0 \\
Cardiovascular diseases & 9 & 11.3 \\
& Other & 8 & 10.0 \\
\hline
\end{tabular}

Correlations of the patients' sex and age with the diagnosis for dysphagia were not statistically significant $(p>0.05)$.

The BBDST-R results and the physicians' diagnosis are presented in Table 3. The prevalence of dysphagia in our sample was $62.5 \%$ (95\% Cl: 50\%-72\%), which was a considerably high percentage based on the data from the previous use of BBDST-R.

Table 3 Results of dysphagia based on the BBDST-R assessment in the present study and the physician's diagnosis.

\begin{tabular}{lllll}
\hline & \multicolumn{2}{l}{ BBDST-R results } & \multicolumn{2}{c}{ Physician's diagnosis } \\
& $\mathrm{N}$ & $\%$ & $\mathrm{~N}$ & $\%$ \\
\hline Negative & 25 & 31.3 & 30 & 37.5 \\
Positive & 55 & 68.8 & 50 & 62.5 \\
Total & 80 & 100.0 & 80 & 100.0 \\
\hline
\end{tabular}

All the BBDST-R items (1-8) presented statistically significant $\phi$ correlations (Table 4) with the diagnosis of the presence or absence of dysphagia. 
Table 4 Correlations of the BBDST-R items with the physician's diagnosis of dysphagia.

\begin{tabular}{|c|c|c|c|c|c|c|c|c|c|}
\hline \multicolumn{10}{|c|}{ Association coefficient } \\
\hline & & $\begin{array}{l}\text { BBDST- } \\
\text { R_1 }\end{array}$ & $\begin{array}{l}\text { BBDST- } \\
\text { R_2 }\end{array}$ & $\begin{array}{l}\text { BBDST- } \\
\text { R_3 }\end{array}$ & $\begin{array}{l}\text { BBDST- } \\
\text { R_4 }\end{array}$ & $\begin{array}{l}\text { BBDST- } \\
\text { R_5 }\end{array}$ & $\begin{array}{l}\text { BBDST- } \\
\text { R_6 }\end{array}$ & $\begin{array}{l}\text { BBDST- } \\
\text { R_7 }\end{array}$ & $\begin{array}{l}\text { BBDST- } \\
\text { R_8 }\end{array}$ \\
\hline Physician's & Phi & $0.649^{* *}$ & $0.477^{* *}$ & $0.467^{* *}$ & $0.507^{* *}$ & $0.507^{* *}$ & $0.511^{* *}$ & $0.306^{* *}$ & $0.323^{* *}$ \\
\hline diagnosis of & Sig. & 0.000 & 0.000 & 0.000 & 0.000 & 0.000 & 0.000 & 0.003 & 0.002 \\
\hline dysphagia & $\mathrm{N}$ & 80 & 80 & 80 & 80 & 80 & 80 & 80 & 80 \\
\hline
\end{tabular}

The $x^{2}$ test and the $\phi$ (Phi) correlation between the results from the BBDST-R tool assessment are presented in Table 5 and Table 6 , respectively. A statistical significance of the $x^{2}$ test and a considerably high value of $\phi$ (Phi) with statistical significance were observed, which demonstrated a strong statistical correlation between the results of the BBDST-R assessment and the physician's diagnosis regarding the presence or absence of dysphagia. Therefore, it could be inferred from Table 5 and Table 6 that the test result was consistent with the medical diagnosis, and the parameters were connected, which is a requisite for a valid test as well as for a proper medical assessment.

Table 5 Chi-Squared test results between the results of the BBDST-R tool assessment.

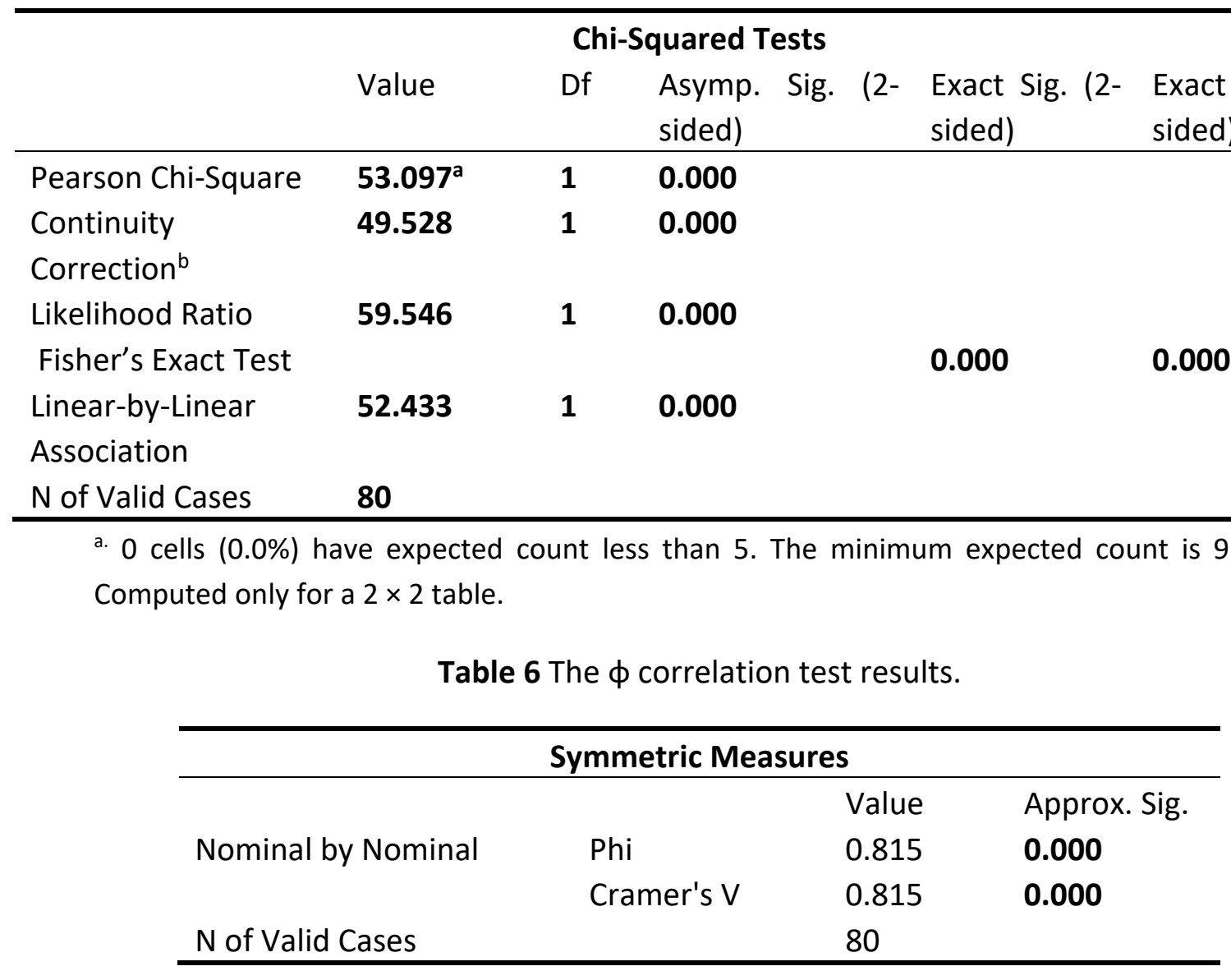

Finally, for the assessment of the diagnostic parameters of the tool, the Sen and Spe indices and the NPV and PPV values were calculated based on the research data presented in Table 7 and Table 8. 
Table 7 Values of the diagnostic accuracy indices.

\begin{tabular}{|c|c|c|c|c|}
\hline \multirow{2}{*}{\multicolumn{5}{|c|}{$\begin{array}{l}\text { Physician's diagnosis for dysphagia * final result } \\
\text { Crosstabulation }\end{array}$}} \\
\hline & & & & \\
\hline & & \multicolumn{2}{|c|}{ BBST-R result } & \multirow{2}{*}{ Total } \\
\hline & & Negative & positive & \\
\hline \multirow{2}{*}{$\begin{array}{l}\text { Physician's diagnosis for } \\
\text { dysphagia }\end{array}$} & No & $24(\mathrm{TN})$ & $6(\mathrm{FP})$ & 30 \\
\hline & Yes & $1(\mathrm{FN})$ & 49 (TP) & 50 \\
\hline Total & & 25 & 55 & 80 \\
\hline
\end{tabular}

Table 8 Overall results for the diagnostic accuracy indices.

\begin{tabular}{llll}
\hline & & \multicolumn{2}{c}{$95 \%$ Confidence Interval } \\
& Estimated value & Lower limit & Upper limit \\
\hline Prevalence & 0.625 & 0.509203 & 0.728684 \\
Sensitivity & 0.98 & 0.879892 & 0.998955 \\
Specificity & 0.8 & 0.608693 & 0.915952 \\
For any particular test, the probability that will be: & \\
Positive & 0.6875 & 0.572854 & 0.783935 \\
Negative & 0.3125 & 0.216065 & 0.427176 \\
For any particular positive test result, the probability that it is: & \\
True positive & 0.890909 & 0.770723 & 0.954865 \\
False positive & 0.109091 & 0.045135 & 0.229277 \\
For any particular negative test result, the probability that it is: & \\
True negative & 0.96 & 0.77677 & 0.997908 \\
False negative & 0.04 & 0.002092 & 0.22323 \\
Likelihood Ratios: [C] & conventional, [W]=Weighted by prevalence & \\
Positive [C] & 4.9 & 2.3928 & 10.034268 \\
Negative [C] & 0.025 & 0.003533 & 0.17688 \\
Positive [W] & 8.166667 & 3.815891 & 17.478089 \\
Negative [W] & 0.041667 & 0.006087 & 0.285239 \\
\hline
\end{tabular}

- Among the 80 cases assessed using the tool, the test was negative for 24 cases, as was the physician's diagnosis for the presence of dysphagia (TN).

- In 6 cases, the test was positive for the presence of dysphagia in contrast to the physician's diagnosis that was negative (FP). Since the physician's diagnosis was considered the benchmark, this finding was considered a false-positive result.

- In just one case, the test result was negative in contrast to the physician's diagnosis of a positive result (FN). False-negative results negatively impact the validity of the tool.

- Finally, for 49 cases, the test result was positive and consistent with the physician's diagnosis for the presence of dysphagia (TP). The tool accurately identified the patients with the concerned condition, which is a desired feature.

In summary, the results were as follows: 


$$
\begin{aligned}
& \text { Sen }=98 \% / 87 \%-99 \%(95 \% \mathrm{Cl}) \\
& \text { Spe }=80 \% / 60 \%-91 \%(95 \% \mathrm{Cl}) \\
& \text { NPV }=89 \% / 77 \%-95 \%(95 \% \mathrm{Cl}) \\
& \text { PPV }=96 \% / 77 \%-99 \%(95 \% \mathrm{Cl})
\end{aligned}
$$

The observed high sensitivity and a high negative predictive value indicated the high validity of the diagnostic tool at the acceptable levels of $>75 \%$ even for $\mathrm{Cl} 95 \%$. The validity of the tool was evaluated in terms of Cronbach's Alpha internal consistency index for its 8 items, which presented an almost excellent value of 0.859 .

In addition, as observed in our results, BBDST-R exhibits a robust performance, as evidenced by the high Sen and NPV for the cut-off score of 1 , implying that 1 abnormal item is sufficient to consider the entire screening result abnormal. Therefore, it was deemed unnecessary to perform tests for other cut-off values. This was consistent with the study reported by Mandysová et al. [15].

\section{Discussion}

The present study is the first complete study that investigated the possibility of using the short version of the BBDST-R tool for the screening of dysphagia in a Greek sample of neurological patients. Specifically, BBDST-R was utilized to assess 80 patients with neurological diseases for the presence or absence of dysphagia followed by a comparison of the results with the corresponding diagnosis provided by a physician.

The literature review revealed the availability of several bedside tools for dysphagia screening, a few of which could be administered by nurses without prior training, a few others require a brief training, while others require further elaborate training to ensure accurate administration. The target groups, administration time, and psychometric properties varied in the different studies reported [25]. In the planning phase of the present study, the literature review did not reveal any existing questionnaire for the bedside screening of dysphagia that was validated in the Greek language, so that the current tool could be validated against it. The researchers, therefore, decided to rely on the physician's diagnosis regarding the presence or absence of dysphagia as the benchmark. The BBDST-R tool was selected for validation due to the advantages of conciseness, the possibility of administration by nurses following brief training, and evidence in favor of its validity and reliability in bedside screening.

The validation of a dysphagia screening tool is necessary because: a) dysphagia is a common symptom of patients with neurological diseases, b) screening in dysphagia is the first step prior to the detailed evaluation of the patient, and c) nurses are the first line of members in the healthcare team to interact with the patients and could, therefore, screen for dysphagia and then refer the patient to the relevant healthcare team member for further examination [25, 26].

The validation and the use of appropriate tools to screen for dysphagia that have already been assessed and used in other countries are necessary [15]. In addition to ensuring the accuracy of the assessment results, the validation allows for comparisons across cultures and aids in the development of common tools for worldwide application. The involvement of nurses in the assessment of dysphagia facilitates collaboration with the entire interdisciplinary team, ultimately reducing the number of dysphagia patients who remain undiagnosed [9]. However, it is also important to establish formal protocols for dysphagia assessment and train the nurses in their application to minimize the time duration for which the patients remain undiagnosed [27]. 
In the present study, the majority of the participants belonged to the age range of 81-90 years. The prevalence of dysphagia in our sample was $62.5 \%$ (95\% Cl: $50 \%-72 \%)$. In another study conducted in a Greek sample [28] with MS, the prevalence ranged between $25.9 \%$ and $44.4 \%$ depending on the assessment tool used.

A strong statistical correlation was observed between the results obtained using BBDST-R and the physician's diagnosis for the presence or absence of dysphagia. The validation of the questionnaires presented a near-perfect score for Cronbach's Alpha (0.859), indicating the reliability of this tool in the Greek setting.

In regard to the $\mathrm{x}^{2}$ test and the $\phi$ (Phi) correlations of the BBDST-R items with the physician's diagnosis of dysphagia, it should be noted that not all items appeared to be strongly correlated with the physician's diagnosis of dysphagia, as items 7 and 8 (Table 4) exhibited weaker correlations. However, in general, the majority of the correlations were satisfactory, proving that the items of the test were examined properly. This was consistent with the overall validity of the tool.

The Sen and the Negative Predictive Value (NPV) indices were calculated to assess the suitability of the tool. High sensitivity (Sen) [Sen = 98\%/87\%-99\% (95\% Cl)] and high negative predictive value (NPV) [NPV $=89 \% / 77 \%-95 \%(95 \% \mathrm{Cl})$ ] were obtained for this tool in the present study, which were comparable to those reported in the original survey [15] [Sen $=95.5 \% / 84.9 \%-98.7 \%(95 \% \mathrm{Cl})$ and NPV $=88.9 \% / 67.2 \%-99.9 \%(95 \% \mathrm{Cl})]$. It is noteworthy that in the original research, the diagnosis was compared with the Flexible Endoscopic Examination of swallowing, which is considered the "gold standard" method, thereby further corroborating the accuracy, validity, and reliability of this tool.

However, PPV and NPV have the following disadvantages: a) they are affected by the prevalence of the disease, b) they cannot be applied to or generalized for the other clinical populations with different admission criteria, and c) they cannot be compared between different diagnostics trials. On the other hand, the sensitivity (Sen) and specificity (Spe) parameters have the following advantages: a) they are not affected by the prevalence of the disease, b) they can be applied to different clinical populations, $c$ ) they can be compared between the studies with different inclusion criteria, and d) they can be used for comparing the diagnostic accuracy between different tests [20, 21].

As with all research, the present study also has certain limitations. First, the present study was designed as a cross-sectional study conducted with a convenience sample. Second, the physician's diagnosis was used as the benchmark for the diagnosis of dysphagia, and the fact that an imaging method (such as the endoscopic examination) was not used as a "gold standard" test is a critical barrier to the generalization of the results and their comparability.

The measured prevalence of dysphagia in our sample was greater than the value of $50 \%$ anticipated during sample size calculation $[\mathrm{P}=62.5 \%(95 \% \mathrm{Cl}: 50 \%-72 \%)]$. However, this finding does not affect the overall robustness of our statistics. Nonetheless, further studies utilizing the BBDST$\mathrm{R}$ tool in other clinical conditions with a larger sample are recommended for corroborating and generalizing the findings of the present study.

\section{Conclusions}

The present study revealed that BBDST-R is a short tool with high sensitivity and a high negative prognostic value for patients with neurological diseases, which does not require extensive staff training for application. Dysphagia screening, being the first step in the elaborate diagnostic 
evaluation of dysphagia, reduces the risk of undiagnosed cases and facilitates interdisciplinary collaboration. BBDST-R could serve as an important tool in the toolkit of Greek nurses, who are hereby advised to use this tool in their daily clinical practice of caring for patients, whether in hospital wards or the community, for a timely and valid evaluation of dysphagia.

\section{Acknowledgments}

Authors would like to acknowledge the Steering Committee of the Postgraduate programme "Neurological Disorders-Evidence Based Nursing" of the University of West Attica for its support, as well as the company Fresenius Kabi Hellas SA for the provision of Thick \& Easy coagulation factor.

\section{Author Contributions}

Sophia Demeneopoulou: conceptualization, methodology, data collection, writing original draft; Marianna Mantzorou: methodology, data interpretation, final manuscript review and editing; Petra Mandysova: data Interpretation, final manuscript review and editing; Nikoletta Margari: methodology, contribution in literature review; Eleni Dokoutsidou: contribution in literature review; Aggeliki Tsiligianni: contribution in literature review; Chrisoula Tsiou: coordination, funding acquisition; Theodoula Adamakidou: conceptualization, supervision of the study, methodology, data interpretation, writing and final editing of the version to be published. All authors read and approved the final manuscript.

\section{Funding}

Funding was received for the publication of this article from the Postgraduate programme "Neurological Disorders-Evidence Based Nursing" of the Nursing Department, University of West Attica, Greece.

\section{Competing Interests}

The authors have declared that no competing interests exist.

\section{References}

1. Peladic NJ, Orlandoni P, Dell'Aquila G, Carrieri B, Eusebi P, Landi F, et al. Dysphagia in nursing home residents: Management and outcomes. J Am Med Dir Assoc. 2019; 20: 147-151.

2. Wirth R, Pourhassan M, Streicher M, Hiesmayr M, Schindler K, Sieber CC, et al. The impact of dysphagia on mortality of nursing home residents: Results from the nutritionDay project. J Am Med Dir Assoc. 2018; 19: 775-778.

3. Park YH, Han HR, Oh BM, Lee J, Park JA, Yu SJ, et al. Prevalence and associated factors of dysphagia in nursing home residents. Geriatr Nurs. 2013; 34: 212-217.

4. Khader FA, Gangadhara SK. Swallowing difficulties among healthy elderly: Prevalence and aetiology. Int J Otorhinolaryngol Head Neck Surg. 2018; 4: 494-498.

5. Madhavan A, LaGorio LA, Crary MA, Dahl WJ, Carnaby GD. Prevalence of and risk factors for dysphagia in the community dwelling elderly: A systematic review. J Nutr Health Aging. 2016; 20: 806-815. 
6. Takizawa C, Gemmell E, Kenworthy J, Speyer R. A systematic review of the prevalence of oropharyngeal dysphagia in stroke, Parkinson's disease, Alzheimer's disease, head injury, and pneumonia. Dysphagia. 2016; 31: 434-441.

7. Malagelada JR, Bazzoli F, Boeckxstaens G, De Looze D, Fried M, Kahrilas P, et al. World gastroenterology organisation global guidelines: Dysphagia-global guidelines and cascades update September 2014. J Clin Gastroenterol. 2015; 49: 370-378.

8. Gallegos C, Brito-De La Fuente E, Clavé P, Costa A, Assegehegn G. Nutritional aspects of dysphagia management. Adv Food Nutr Res. 2017; 81: 271-318.

9. Werner H. The benefits of the dysphagia clinical nurse specialist role. J Neurosci Nurs. 2005; 37 : 212-215.

10. Dondorf K, Fabus R, Ghassemi AE. The interprofessional collaboration between nurses and speech-language pathologists working with patients diagnosed with dysphagia in skilled nursing facilities. J Nurs Educ Pract. 2016; 6: 17-20.

11. Jiang JL, Fu SY, Wang WH, Ma YC. Validity and reliability of swallowing screening tools used by nurses for dysphagia: A systematic review. Ci Ji Yi Xue Za Zhi. 2016; 28: 41-48.

12. Scottish Intercollegiate Guidelines Network. Management of patients with stroke: Identification and management of dysphagia. A national clinical guideline. Edinburgh: Scottish Intercollegiate Guidelines Network; 2010.

13. Herdman HT, Kamitsuru S. NANDA international nursing diagnoses: Definitions \& classification 2018-2020. New York: Thieme; 2017. pp.173.

14. Mandysová P, Fusek J, Tsiou C, Plakas S. A cross-border educational session promoting nursing dysphagia screening in Greece: A pilot study. Kontakt. 2016; 18: e56-e63.

15. Mandysová $P$, Ehler E, Škvrňáková J, Černý M, Bártová I, Pellant A. Development of the Brief Bedside Dysphagia Screening Test-Revised: A cross-sectional Czech study. Acta Med. 2015; 58: 49-55.

16. Mandysová $P$, Trundová $H$, Ehler E. Inter-rater agreement of the brief bedside dysphagia screening test-revised in patients with stroke. Cent Eur J Nurs Midwifery. 2016; 7: 412-419.

17. Mandysova P, Škvrňáková J, Ehler E, Černý M. Development of the brief bedside dysphagia screening test in the Czech Republic. Nurs Health Sci. 2011; 13: 388-395.

18. Trust MO. Trust introduces new translation criteria. Med Outcomes Trust Bull. 1997; 5: 1-4.

19. Altman DG, Bland JM. Statistics notes: Diagnostic tests 1: Sensitivity and specificity. BMJ. 1994; 308: 1552.

20. Glas AS, Lijmer JG, Prins MH, Bonsel GJ, Bossuyt PM. The diagnostic odds ratio: A single indicator of test performance. J Clin Epidemiol. 2003; 56: 1129-1135.

21. Šimundić AM. Measures of diagnostic accuracy: Basic definitions. EJIFCC. 2009; 19: 203-211.

22. Molina M. A never-ending story [Internet]. 2014. Available from: https://www.cienciasinseso.com/en/diagnostic-odds-ratio/.

23. Flahault A, Cadilhac M, Thomas G. Sample size calculation should be performed for design accuracy in diagnostic test studies. J Clin Epidemiol. 2005; 58: 859-862.

24. Chu H, Cole SR. Sample size calculation using exact methods in diagnostic test studies. J Clin Epidemiol. 2007; 60: 1201-1202.

25. Fedder WN. Review of evidenced-based nursing protocols for dysphagia assessment. Stroke. 2017; 48: e99-e101. 
26. Campbell GB, Carter T, Kring D, Martinez C. Nursing bedside dysphagia screen: Is it valid? J Neurosci Nurs. 2016; 48: 75-79.

27. Hines S, Wallace K, Crowe L, Finlayson K, Chang A, Pattie M. Identification and nursing management of dysphagia in individuals with acute neurological impairment (update). Int J Evid Based Healthc. 2011; 9: 148-150.

28. Printza A, Triaridis S, Kalaitzi M, Nikolaidis I, Bakirtzis C, Constantinidis J, et al. Dysphagia prevalence, attitudes, and related quality of life in patients with multiple sclerosis. Dysphagia. 2019; 35: 677-684.

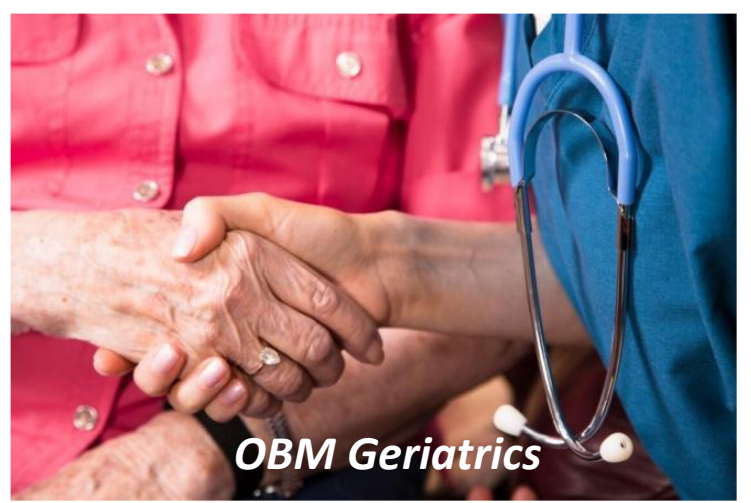

Enjoy $O B M$ Geriatrics by:

1. Submitting a manuscript

2. Joining in volunteer reviewer bank

3. Joining Editorial Board

4. Guest editing a special issue

For more details, please visit: http://www.lidsen.com/journals/geriatrics 